\title{
Early diagnosis of trisomy 21, trisomy 18 and trisomy 13 using nuchal translucency thickness and ductus venosus blood flow waveform in West China
}

\author{
YING TANG ${ }^{1,2}$, HONG LUO ${ }^{1,2}$, DEZHI MU ${ }^{2,3}$, TAIZHU YANG ${ }^{1,2}$, QI ZHU ${ }^{1,2}$, \\ FAN YANG ${ }^{1,2}$ and GUANJIAN LIU ${ }^{4}$ \\ ${ }^{1}$ Department of Ultrasonography, West China Women and Children Hospital Affiliated to Sichuan University; \\ ${ }^{2}$ Key Laboratory of Birth Defects and Related Disease of Women and Children, Ministry of Education; \\ Departments of ${ }^{3}$ Pediatrics and ${ }^{4}$ Evidence-Based Medicine and Clinical Epidemiology, \\ West China Hospital, Sichuan University, Chengdu, Sichuan 610041, P.R. China
}

Received March 28, 2018; Accepted November 16, 2018

DOI: $10.3892 / \mathrm{mmr} .2018 .9756$

\begin{abstract}
Abnormal nuchal translucency (NT) thickness and ductus venosus (DV) blood flow have been associated with trisomy 21, 18 and 13. However, the association of abnormal DV with these aneuploidies varies among clinical studies. The present study examined the possibility of using NT combined with DV for the early diagnosis of the three aforementioned aneuploidies in the Western Chinese population, focusing on three aspects: Biological pathway analysis, theoretical statistical analysis and clinical data analysis from 1,962 first-trimester pregnant women from Western China. The pathway and statistical analyses performed suggested the reliability of integrating NT and DV in the prediction of the three aneuploidies. The clinical data analysis suggested that integrating NT and DV, compared with NT alone, resulted in increased predictive power (34.09 vs. $22.45 \%$ ), better rejection probability ( 0.21 vs. $0.44 \%)$, increased specificity ( 96.71 vs. $94.07 \%)$ and increased sensitivity (89.47 vs. $80.49 \%)$. The present results suggested the reliability of integrating NT and DV for the early diagnosis of trisomy 21, 18 and 13 for the Western Chinese population. The present results provided novel statistical analyses to the field of prenatal diagnosis in the Western Chinese population.
\end{abstract}

\section{Introduction}

The ductus venosus (DV) is a blood vessel specific to the fetal period and belongs to an important blood circulatory network.

Correspondence to: Dr Hong Luo, Department of Ultrasonography, West China Women and Children Hospital Affiliated to Sichuan University, 20 Renmin South Road 3rd Section, Chengdu, Sichuan 610041, P.R. China

E-mail: h.luo@gousinfo.com

Key words: trisomy 21, trisomy 18, trisomy 13, nuchal translucency thickness, ductus venosus blood flow
DV blood flow allows oxygenated blood from the placenta to bypass the liver (1). In 1991, using ultrasound, Kiserud et al (2) observed the DV in fetuses between middle and late pregnancy, investigating the spectrum of the blood flow. A previous study investigated the association between fetal DV and chromosomal abnormalities in the first trimester of pregnancy (8-14 weeks) (3).

The most common aneuploidy-associated syndromes are: Down syndrome caused by trisomy 21 (T21), Patau syndrome, caused by trisomy 13 (T13), and Edwards syndrome, caused by trisomy 18 (T18) (4). Nuchal translucency (NT) thickness is an established method for the prediction of all three diseases (5-7). A previous study suggested that abnormal DV may be the cause for the increased NT thickness in the case of aneuploidy (8). DV assessment may improve early detection of congenital heart defects compared with NT measurement alone (9). In addition, Maiz et al (10) demonstrated that abnormal DV was associated with T21, T18 or T13. Maiz et al (10) observed that $\sim 7.7 \%$ of fetuses presented abnormal DV, and these fetuses exhibited a thicker NT. Therefore, NT and DV may be used for the early diagnosis of trisomy.

Although multiple previous studies tested the feasibility of combining NT and DV for the prediction of the three trisomies (T21, T18 and T13), the results were variable (Table I). The association between abnormal DV and T21 varied between 38.3 and $92.1 \%$, depending on the population examined $(11,12)$. Furthermore, previous studies tested the reliability of integrating NT and DV for the diagnosis of T21 in the Chinese population (13), and in the diagnosis of the other two trisomies (T18 and T13) (10). In the present study, a multi-level analysis was performed to test the reliability of combining NT with DV for the prediction of T21, T18 and T13 in the Western Chinese population. The present study aimed to provide novel statistical analyses to the field of prenatal diagnosis in the Western Chinese population.

\section{Materials and methods}

Study design. Pathway analysis was conducted to derive association data from literature. Subsequently, Bayes' 
theorem-based statistical analysis was performed to calculate the conditional probabilities. The formulas based on Bayes' theorem describe how to calculate the probability of an event, based on its association with another event (14). Additionally, a statistical analysis was performed based on clinical data from 1,962 first-trimester pregnant women from Western China.

Pathway analysis. Data regarding the associations between diseases and genes, and among various diseases were obtained from the Pathway Studio (www.pathwaystudio.com; version 12.0) (15) generated by the MedScan ${ }^{\circledR}$ text-mining tool (16). The Pathway Studio database contains all abstracts from PubMed and millions of full-text articles from journals belonging to Elsevier B.V. (Amsterdam, The Netherlands).

Statistical estimation. Besides sensitivity and specificity, the present study aimed to use Bayes' theorem to estimate the disease predictive power and disease rejection power considering a thickened NT, an abnormal DV or the two parameters combined.

Sensitivity using DV, NT or the two parameters combined. The sensitivity using $\mathrm{X}$ to predict $\mathrm{Y}$ is described as a conditional probability $\mathrm{p}(\mathrm{X} / \mathrm{Y})$, which is calculated using Bayes' theorem, (Equation i).

$$
\mathrm{p}(\mathrm{X} / \mathrm{Y})=\mathrm{p}(\mathrm{X} / \mathrm{Y}) / \mathrm{P}(\mathrm{Y})
$$

Where $\mathrm{X}=\mathrm{DV}$, NT or $(\mathrm{DV} I \mathrm{NT})$; $\mathrm{Y}=\mathrm{T} 21, \mathrm{~T} 18, \mathrm{~T} 13$ and $\mathrm{T}$. Where (DVIINT) represents one of the combinations of DV or NT, and T represents any of the three trisomies examined.

Specificity using DV/NT or the two combined. The specificity of using $\mathrm{X}$ to predict $\mathrm{Y}$ is described by the conditional probability (Equation ii).

$$
p(\bar{X} / \bar{Y})=p(\bar{X} / \bar{Y}) / P(\bar{Y})
$$

Where $\bar{X}=\overline{D V}, \overline{N T}$ or $(\overline{N T \& D V}) ; \bar{Y}=\overline{T 21}, \overline{T 18}, \overline{T 13}$ or $\bar{T}$.

Predictive power using DVINT or the two combined. According to Bayes' theory, the predictive power of using $\mathrm{X}$ to predict $\mathrm{Y}$ is calculated with Equation iii. The predictive power defines the probability of Y considering that X occurred.

$$
p(Y / X)=p(X Y) / P(X)
$$

Where $\mathrm{X}=\mathrm{DV}, \mathrm{NT}, \mathrm{DV} \& \mathrm{NT}$ and $\overline{D V} \& \overline{N V} ; \mathrm{Y}=\mathrm{T} 21, \mathrm{~T} 18, \mathrm{~T} 13$ and $\mathrm{T}$. The predictive power is associated with the reliability of using $\mathrm{X}$ as an indicator for the prediction of $\mathrm{Y}$.

Rejection power using DV/NT or the two combined. The rejection power of using $\mathrm{X}$ to predict $\mathrm{Y}$ is expressed as a conditional probability (Equation iv), which indicates the probability of $\mathrm{Y}$ based on the fact that $\mathrm{X}$ did not occur.

$$
\mathrm{p}(\mathrm{Y} / \bar{X})=\mathrm{p}(\bar{X} \mathrm{Y}) / \mathrm{P}(\bar{X})
$$

Where $\bar{X}=\overline{D V}, \overline{N T}$ and $(\overline{D V} \& \overline{N T}) ; \mathrm{Y}=\mathrm{T} 21, \mathrm{~T} 18, \mathrm{~T} 13$ and $\mathrm{T}$. The conditional probability $\mathrm{p}(\mathrm{Y} / \bar{X})$ is negatively associated with the rejection power. A high rejection power indicates high reliability of using $\mathrm{X}$ as an indicator for the prediction of $\mathrm{Y}$.

Clinical data. A total of 2,098 pregnant women were recruited for the present study. The data obtained were categorized into two groups $\left(N_{1}=960\right.$ and $\left.N_{2}=1,138\right)$. In the first group, NT thickness and DV blood flow were examined. In the second group, only NT thickness was measured. The inclusion criteria were: Single pregnancy, early fetal NT examination, 11-13 weeks pregnancy and fetal crown-rump length (CRL): 45-84 mm. Patients were recruited between January 2010 and December 2014 in The West China Women and Children Hospital Affiliated to Sichuan University (Chengdu, China). The exclusion criteria were: Severe organ dysfunction, lack of follow-up, fetal chromosomal abnormalities other than the three trisomies examined in the present study, severe fetal abnormalities and miscarriage. The present study was approved by The Ethics Committee of Sichuan University. Informed consent was signed by all patients. Following removal of the patients meeting the exclusion criteria, the first group consisted of 903 patients and the second group consisted of 1,059 patients.

NT and DV data acquisition. For the two groups, the morphology of the fetus in the first trimester was analyzed and NT was measured. The ultrasonography test was performed by four physicians in the hospital. Ultrasound examination was performed in $10 \mathrm{~min}$. The frequency of the probe was 3.5-5.0 MHz. The ultrasound machines used were: Voluson 730 (GE Healthcare, Chicago, IL, USA), Sequoia 512 (Siemens AG, Munich, Germany) and IU22 (Philips Healthcare, Andover, MA, USA). The thermal index was $<0.8$ and the mechanical index was $<0.77$. Routine fetal examinations (including number of fetuses, CRL, placenta and amniotic fluid) were conducted to confirm the gestational age. The ultrasound results were recorded prior to NT measurement. NT measurements were performed according to The Fetal Medicine Foundation system (17). NT was considered abnormal when the NT was $\geq 3 \mathrm{~mm}$ or when fetal cystic hygroma was detected.

The DV waveforms were measured in the first group $\left(N_{1}=960\right)$, and the NT was additionally measured. The DV blood flow was directed into the atrium and the flow velocity detected was higher compared with the peripheral vein blood flow. The sampling frame of $2 \mathrm{~mm}$ was placed at the beginning of the catheter. The sound beam and blood flow direction were close to $0^{\circ}$, using a Doppler correction angle $<60^{\circ}$. Doppler velocity curve of the venous pulse was examined when the fetus was in resting state. The average velocity during five cardiac cycles was automatically calculated and recorded by the built-in software of the instrument. The total time of detection of the DV was $<3 \mathrm{~min}$. In the case of normal DV flow, all waves indicated the forward direction of blood flow (Fig. 1A). These waves include the ventricular contraction wave, the ventricular diastolic and the atrial contraction wave (A wave). DV blood flow was considered abnormal when the absence or the reversal of the A-waves were detected (Fig. 1B).

Other examinations and follow-up. Pregnant women exhibiting the following fetal chromosomal abnormality risk 
Table I. Previous studies demonstrating various co-occurrence rates of abnormal DV and trisomies.

\begin{tabular}{|c|c|c|c|c|c|c|}
\hline Author, year & $\mathrm{n}$ & $\mathrm{T} 21$ & T18 & $\mathrm{T} 13$ & Population examined & (Refs.) \\
\hline Maiz et al, 2009 & 19,800 & $81 / 122(66.4 \%)$ & $21 / 36(58.3 \%)$ & $11 / 20(55 \%)$ & British & (10) \\
\hline Prefumo et al, 2005 & 572 & 18/47 (38.3\%) & - & - & British & (11) \\
\hline Matias et al, 1998 & 486 & $35 / 38(92.1 \%)$ & $12 / 12(100 \%)$ & $5 / 7(71.4 \%)$ & Mixed & (12) \\
\hline Hsiao et al, 2014 & 20,586 & $12 / 25(48.0 \%)$ & - & - & Chinese, Taiwanese & (13) \\
\hline Murta et al, 2002 & 372 & $18 / 18(100 \%)$ & $1 / 1(100 \%)$ & $2 / 2(100 \%)$ & Brazilian & $(18)$ \\
\hline Zoppi et al, 2002 & 325 & $14 / 20(70 \%)$ & $6 / 7(85.7)$ & $1 / 1(100 \%)$ & Italian & (19) \\
\hline Borrell et al, 2003 & 3,382 & $36 / 48(75 \%)$ & $17 / 24(71 \%)$ & - & Spanish & $(20)$ \\
\hline Antolin et al, 2001 & 924 & $5 / 7(71.4 \%)$ & $3 / 3(100 \%)$ & - & Spanish & $(21)$ \\
\hline Toyama et al, 2004 & 1,097 & $5 / 7(71.4)$ & $3 / 5(60 \%)$ & $1 / 1(100 \%)$ & Brazilian & $(22)$ \\
\hline Stressig et al, 2011 & 3,648 & $5 / 35(14.3 \%)$ & - & - & German & (23) \\
\hline Wagner et al, 2016 & 4,641 & - & $32 / 40(80 \%)$ & $8 / 13(61.5 \%)$ & German & (24) \\
\hline
\end{tabular}

DV, ductus venosus; $\mathrm{T}$, trisomy.
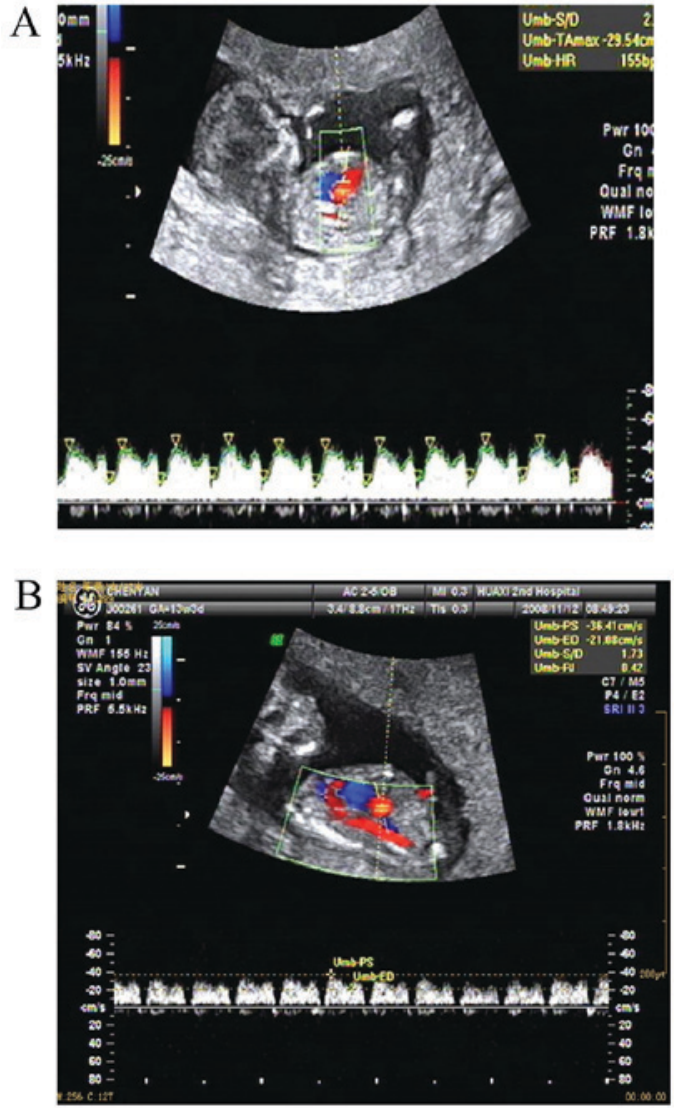

Figure 1. DV flow assessment. Fetuses with (A) normal and (B) abnormal DV during the 13th week of pregnancy. DV, ductus venosus.

factors: Thickened NT, >35 years old, serological abnormalities, adverse reproductive history, adverse pregnancy exposure history and family history of genetic disease, were recommended to undergo a karyotype test following amniocentesis or cordocentesis. A follow-up study was conducted by a team composed of an obstetrician, a pediatrician and ultrasonologists, with the aim to analyze the results of the karyotype test, neonatal examination and, in case of miscarriage, examination of the fetus following labor induction. Communication by telephone, face-to-face interviews and hospital records were used to conduct the follow-up study.

\section{Results}

Pathway analysis. The pathway analysis suggested that three genes, jagged 1, pappalysin 1 (PAPPA) and 5'-nucleotidase ecto, and nine diseases were associated with all three trisomies (T21, T18 and T13). Furthermore, certain genes and diseases identified were associated with NT and DV (Fig. 2). Specifically, NT and DV were associated with congenital heart defects and with PAPPA. The present results suggested that NT and DV are associated with all three trisomies, which suggested the possibility of using NT with DV as a clinical indicator for the diagnosis of T21, T18 and T13.

Statistical analysis. Mathematical statistical analysis using Bayes' theorem suggested that combining DV and NT may lead to increased specificity and sensitivity. However, to improve the prediction and rejection power using the combination of DV and NT, certain conditions are required, which were described above in the 'Prediction power' and 'Rejection power' sections. $X_{1}$ was selected to indicate the occurrence of abnormal NT, and $X_{2}$ to indicate the occurrence of abnormal DV. Y indicates the occurrence of any trisomy among T13, T18 and T21.

Improved sensitivity. As $p((N T \| D V) \& Y) \geq p(N T \& Y)$, i.e., $p\left(\left(X_{1} \| X_{2}\right) \& Y\right) \geq p\left(X_{2} \& Y\right)$, subsequently,

$$
p\left(\left(X_{1} \| X_{2}\right) / Y\right)=\frac{p\left(X_{1} \| X_{2} \& Y\right)}{P(Y)} \geq \frac{p\left(X_{2} Y\right)}{P(Y)}=p\left(X_{2} / Y\right)
$$

Where $p\left(\left(X_{1} \| X_{2}\right) / Y\right)$ refers to the sensitivity of using $N T$ and $D V$ to predict $\mathrm{Y}$ (e.g., when one of the conditions of NT or DV occurs, Y occurs); $p\left(X_{2} / Y\right)$ refers to the sensitivity of using $N T$ alone to predict $Y$. Equation $v$ suggested that combining NT with DV may lead to an improved sensitivity in predicting the occurrence of trisomies. 
Table II. A total of 41 cases of trisomies from the two groups of 1,962 participants.

\begin{tabular}{|c|c|c|c|c|c|c|}
\hline \multirow[b]{2}{*}{$\mathrm{T}$} & \multicolumn{3}{|c|}{ Group $1\left(N_{1}=903\right)$} & \multicolumn{2}{|c|}{ Group $2\left(N_{2}=1,059\right)$} & \multirow[b]{2}{*}{ Total } \\
\hline & DV\&NT & $\mathrm{DV} \& \overline{N T}$ & $\overline{D V} \& \overline{N T}$ & NT & $\overline{N T}$ & \\
\hline $\mathrm{T} 21$ & 10 & 1 & 2 & 13 & 4 & 30 \\
\hline $\mathrm{T} 18$ & 3 & 1 & - & 3 & - & 7 \\
\hline $\mathrm{T} 13$ & 2 & - & - & 2 & - & 4 \\
\hline Any & 15 & 2 & 2 & 18 & 4 & 41 \\
\hline
\end{tabular}

DV, ductus venosus; NT, nuchal translucency; T, trisomy.

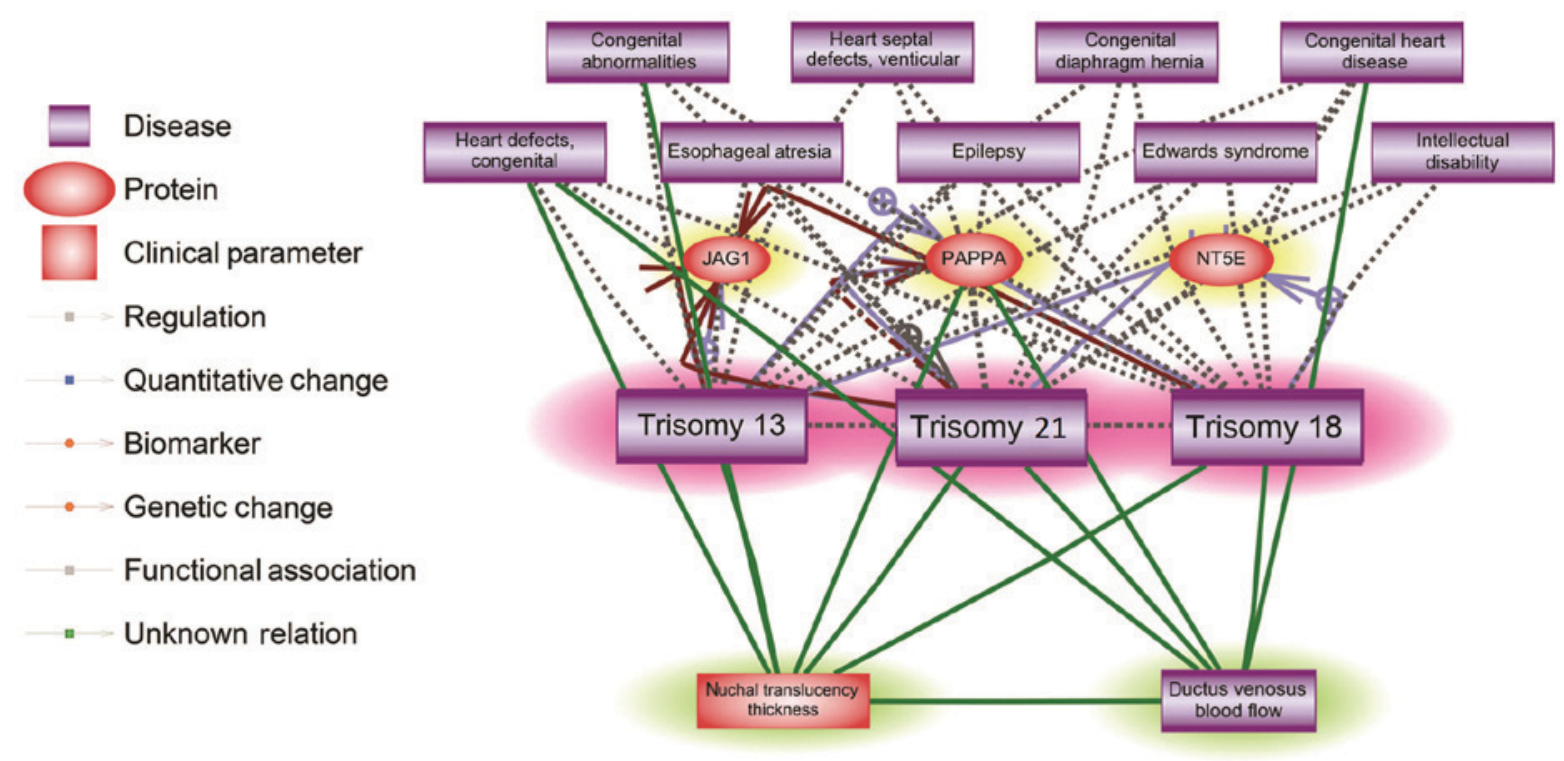

Figure 2. Diagram of the functional network demonstrating possible associations between NT and DV, and T21, T18 and T13. Network was built using Pathway Studio software and it contains three common genes (highlighted in yellow), nine common associated diseases (not highlighted), and two clinical parameters: NT and DV (highlighted in green). DV, ductus venosus; NT, nuchal translucency; T, trisomy. For detailed description of the network, please refer to the supplementary file (https://figshare.com/s/8a2728cf0d42502eece4).

Improved specificity. As $p(\overline{N T \& D V} \& \bar{Y}) \geq p(\overline{N T \& Y})$, i.e., $\mathrm{p}\left(\bar{X}_{1} \& X_{2} \& \bar{Y}\right) \geq p\left(\bar{X}_{2} \& \bar{Y}\right)$, subsequently,

$$
p\left(\overline{X_{1} \& X_{2}} / \bar{Y}\right)=\frac{p\left(\overline{X_{1} \& X_{2}} \bar{Y}\right)}{P(\bar{Y})} \geq \frac{p\left(\overline{X_{2}} \bar{Y}\right)}{P(\bar{Y})}=p\left(\overline{X_{2}} / \bar{Y}\right)
$$

Where $p\left(\bar{X}_{1} \& \bar{X}_{2} / \bar{Y}\right)$ is the specificity of using NT with DV to predict $\mathrm{Y} ; p\left(\bar{X} \bar{X}_{2} / \bar{Y}\right)$ is the specificity for using $N T$ alone to predict $Y$. Equation vi suggested that, theoretically, combining NT and DV may lead to an improved specificity in predicting the occurrence of trisomies.

Prediction and rejection power. The prediction probability $p\left(Y / X_{1}\right)$ describes the probability of $Y$ based on the fact that $X_{1}$ occurs. The rejection probability $p\left(Y / \bar{X}_{1}\right)$ describes the probability of $Y$ based on the fact that $X_{1}$ does not occur. In order to improve the reliability of the diagnosis, increased predictive power and decreased rejection probability are required. If $p\left(Y / X_{1} \bar{X}_{2}\right)<p\left(Y / X_{1} X_{2}\right)$, using $X_{1}$ with $X_{2}$ can lead to improved predictive power and rejection power compared with $X_{1}$ alone. However, clinical data are required to estimate and test the conditional probabilities derived by Bayes' theorem.

Clinical results. The occurrence of each trisomy in different cases are presented in Table II. The first group was composed of 903 subjects. Among these subjects, 58 presented DV abnormality, 63 exhibited thickened NT, and 44 presented abnormal DV and thickened NT, concomitantly. The second group was composed of 1,059 patients. A total of 84 patients exhibited thickened NT. The statistical analyses associated with DV were performed considering data from the first group. Regarding NT, data from the two groups were considered. The results of the statistical analyses performed are presented in Table III. Collectively, the sensitivity, specificity and the predictive power were calculated, considering DV alone, NT alone or combining DV and NT.

The results presented in Table III suggested that, in order to predict the occurrence of trisomies, combining DV and NT resulted in increased sensitivity and specificity compared with one indicator alone. Accordingly, combining the two indicators 
Table III. Probabilities calculated using the two datasets.

\begin{tabular}{lccccc}
\hline Statistics values & Cases & T21, $\%$ & T18, & T13, $\%$ & Any T, \% \\
\hline Sensitivity & $\mathrm{p}(\mathrm{DV} / \mathrm{T})$ & 84.62 & 100.00 & 100.00 & 89.47 \\
& $\mathrm{p}(\mathrm{NT} / \mathrm{T})$ & 76.67 & 85.71 & 100.00 & 80.49 \\
\multirow{3}{*}{ Specificity } & $\mathrm{p}((\mathrm{NT} \& \mathrm{DV}) / \mathrm{T})$ & 84.62 & 100.00 & 100.00 & 89.47 \\
& $\mathrm{p}(\overline{D V} / \bar{T})$ & 94.81 & 93.91 & 93.77 & 95.35 \\
Predictive Power & $\mathrm{p}(\mathrm{NT} / \bar{T})$ & 93.58 & 92.79 & 92.7 & 94.07 \\
& $\mathrm{p}((\overline{N T \& D V}) / \bar{T})$ & 96.27 & 95.36 & 95.32 & 96.71 \\
& $\mathrm{p}(\mathrm{T} / \mathrm{DV})$ & 18.97 & 6.90 & 3.45 & 29.31 \\
Rejection Power & $\mathrm{p}(\mathrm{T} / \mathrm{NT})$ & 15.65 & 4.08 & 2.72 & 22.45 \\
& $\mathrm{p}(\mathrm{T} /(\mathrm{NT} \& \mathrm{DV}))$ & 22.73 & 6.82 & 4.56 & 34.09 \\
& $\mathrm{p}(\mathrm{T} / \overline{D V})$ & 0.332 & 0 & 0 & 0.22 \\
& $\mathrm{p}(\mathrm{T} / \overline{N T})$ & 0.386 & 0.055 & 0 & 0.44 \\
& $\mathrm{p}(\mathrm{T} /(\overline{N T} \& \overline{D V}))$ & 0.27 & 0 & 0 & 0.21 \\
\hline
\end{tabular}

Other probabilities estimated $p(\underline{D V})=6.42 \%, p(N T)=7.49 \%$ and $p(\mathrm{DV} \& \mathrm{NT})=4.87 \%$. DV, ductus venosus; $\mathrm{NT}$, nuchal translucency; $\mathrm{T}$, trisomy; $\mathrm{p}[\mathrm{T} /(N T \& \overline{D V})]=0.80 \% ; \mathrm{p}[\mathrm{T} /(\overline{N T} \& D V)]=14.29 \%$.
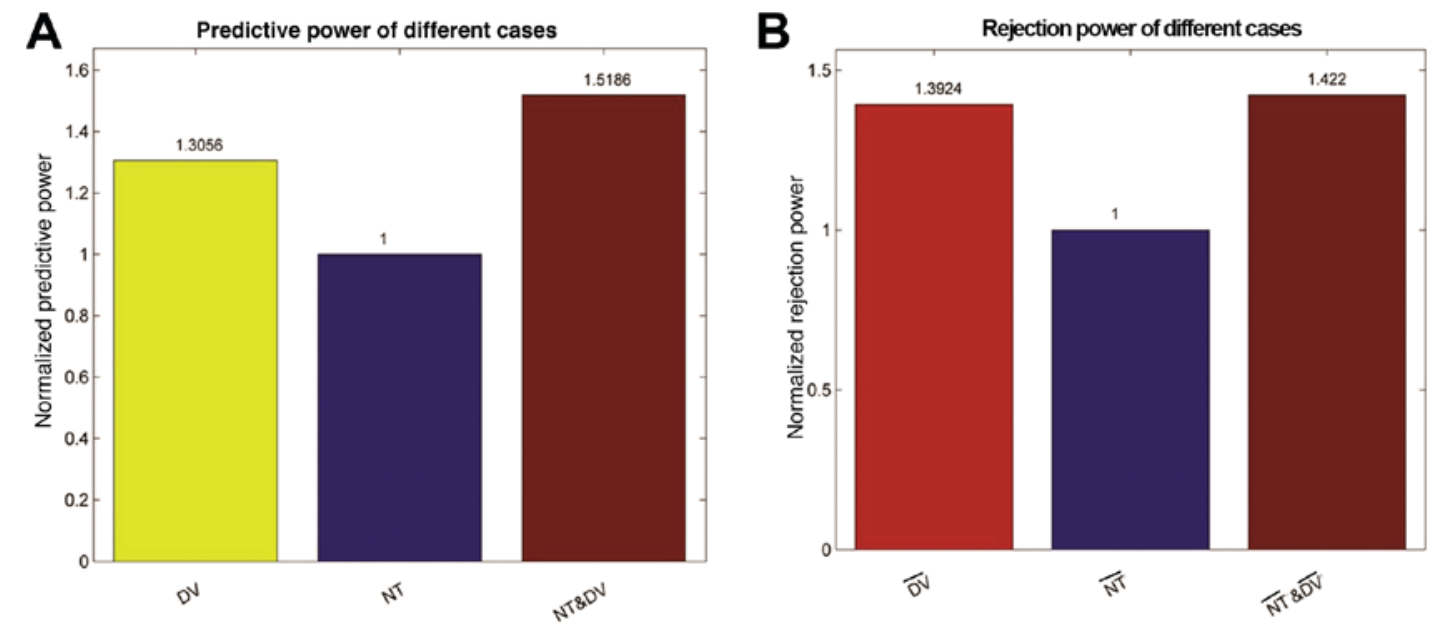

Figure 3. Comparison of the diagnosis power using three parameters: DV, NT and DV with NT. (A) Normalized predictive power: All the prediction probabilities were normalized to NT alone. (B) Normalized rejection power: The reciprocal rejection probabilities were normalized to NT alone. Reciprocal rejection probability is presented since the rejection probability and the rejection power are inversely associated. The color of the bars does not reflect the value of the variables but is merely to increase the visibility of the figure. DV, ductus venosus; NT, nuchal translucency.

increased the predictive power by over $50 \%$, and the rejection power increased by over $40 \%$, as presented in Fig. 3 .

\section{Discussion}

While NT has been well accepted as prospective screening indicator of chromosomal abnormalities, recently, DV wave form has also been suggested as an additional clinical maker for the early trisomy diagnosis (18). However, previous studies considering various populations led to contrasting results, and the number of previous studies analyzing the Chinese population is limited (3-7,10-13). The present study investigated the reliability of combining DV and NT for the early diagnosis of the three most common trisomies in Western China: T21, T18 and T13. The analyses performed were based on: Investigation of previous literature, theoretical statistical analysis and analysis of clinical data.
The pathway analysis suggested that DV and NT were associated with multiple risk factors, including diseases and gene dysregulations that were associated with the three trisomies, as shown in Fig. 2. Note that for each relation (edge) presented in Fig. 2, there were one or more supporting references. In total, there were about 825 references supporting the relationships presented in Fig. 2 (please see 'Additional data' available at https://figshare.com/s/8a2728cf0d42502eece4). In addition to the directly reported relationships $(19,20)$, other evidences were also presented in the pathway (Fig. 2) in favor of the rationality of using both NT and DV for the detection of chromosomal abnormalities. Previous studies suggested that NT thickness, DV abnormalities and the expression levels of PAPP-A may be considered in the diagnosis of chromosomal abnormalities to increase the detection rate and decrease the rate of false positives $(21,22)$. Both NT screening and DV have been suggested 
in the detection of congenital heart defects (21). On the other hand, fetal heart diseases have been strongly associated with T21 (22), T18 and T13 (23). That both NT and DV were clinical markers for trisomy-related diseases (e.g., congenital heart defects) $(18,24,25)$ may partially explain the rationality of using NT and DV as clinical markers for the early diagnosis of all three trisomies. Mathematical statistical analyses suggested that combining NT with DV may improve the prediction sensitivity and specificity in the diagnosis of trisomies. Furthermore, for the purpose of diagnosis, the predictive power and rejection power are important factors to consider. The predictive power describes the occurrence probability of a trisomy when at least one risk factor was detected. The rejection power represents the occurrence probability of any trisomy when no risk factors were detected. In order to make an accurate diagnosis, it is important to increase the prediction probability and decrease the rejection probability. Unlike sensitivity and specificity, the analysis of predictive power and rejection power requires validation using clinical data.

Clinical data from 1,962 Western Chinese patients were used to calculate sensitivity, specificity, and prediction and rejection probabilities. The present results suggested that the combination of NT and DV led to improved sensitivity and specificity. Specifically, when abnormal NT and DV were observed concomitantly, the occurrence probability of any trisomy increased, compared with NT alone $34.09 \%$ vs. $22.45 \%$ ). In case of normal NT and DV the occurrence probability was decreased by over $50 \%$ (0.205\% vs. $0.441 \%)$. Additionally, when only NT or DV presented abnormalities, the occurrence probability of trisomy was $0.80 \%$ in the case of thickened NT alone and $14.29 \%$ in the case of abnormal DV alone (Table III). Furthermore, compared with NT or DV alone, combining NT and DV resulted in increased predictive power and rejection power.

Additional statistical parameters were estimated, including overall abnormal DV rate $(6.4 \%)$, overall abnormal NT rate (7.49\%) and concomitant abnormal DV and NT rate $(4.87 \%)$. Notably, abnormal NT alone led to various occurrence rates of T21, T18 and T13 (76.67, 85.71 and 100\%, respectively). The present estimated values are consistent with previous studies $(8,10,22)$. Additionally, the clinical results suggested that fetuses with abnormal DV presented a significant thicker NT (3.9 mm) compared with fetuses with normal DV $(1.4 \mathrm{~mm})$, consistent with the hypothesis that abnormal DV is associated with thickened NT (3). Notably, the differences observed may be specific of the population of patients considered; the present study only analyzed Western Chinese women. In the present study, the sensitivity of T21 prediction using only DV ( 84.6\%) was different from the previous study of Hsiao et al (13) ( $48.0 \%)$, where a Southern Chinese population was considered. In addition to the association between T21 and abnormal DV, the possibility of using abnormal DV to predict the occurrence of T18 and T13 was examined. This possibility was not investigated by previous studies analyzing the Chinese population, to the best of the authors' knowledge. Therefore, the present study may provide novel statistical analyses to the field of prenatal diagnosis in the Western Chinese population.

Although combining NT and DV may significantly increase the predictive power, the prediction probability remains insufficient (34.09\%). Therefore, besides NT and DV, other factors may exist that influence the occurrence of trisomy-associated diseases. In the present study, normal NT (and normal DV for group one) were detected for two fetuses with aneuploidy from group one and five fetuses from group two. In case of normal NT and DV, the aneuploidy may be due to the advanced reproductive age or serological abnormalities of the mother, as recorded in the clinical data. Therefore, further studies analyzing a number of clinical datasets are required in order to identify additional clinical parameters, besides NT and DV, that may be associated with the occurrence of chromosomal aneuploidy.

The present study is based on a multi-level analysis, and suggested that, without conducting further screening and thus increasing the cost of the analysis, combining DV and NT may increase the predictive power in identifying trisomies (T21, T18 and T13), leading to an improvement in prenatal diagnoses. The present results may provide insight into the field of prenatal diagnosis of chromosome abnormalities in the Western Chinese population.

\section{Acknowledgements}

The authors would like to thank Ms. Lydia Manor from Children's National Health Hospital for providing the Pathway Studio association data analysis.

\section{Funding}

The present study was supported by The Science and Technology Bureau of Chengdu, The Huimin Technology R\&D Project (grant no. 2014-HM01-00049-SF; China) and The Science and Technology Department of Sichuan Province, The Application of Basic Science Project (grant no. 2017JY0263; China).

\section{Availability of data and materials}

The datasets used and analyzed during the current study are available from the corresponding author on reasonable request. The additional data generated during the current study are available in the Figshare repository (https://figshare. com/s/8a2728cf0d42502eece4).

\section{Authors' contributions}

YT and HL contributed to the data collection and data analysis. YT, HL, DM and GL contributed the study design and manuscript development. TY, QZ and FY contributed to the clinical data collection and manuscript development.

\section{Ethics approval and consent to participate}

The present study was approved by The Ethics Committee of Sichuan University (Chengdu, China). Informed written consent was obtained from all individual participants included in the present study.

\section{Patient consent for publication}

All patients provided consent for publication. 


\section{Competing interests}

The authors declare that they have no competing interests.

\section{References}

1. Mavrides E, Moscoso G, Carvalho JS, Campbell S and Thila-ganathan B: The human ductus venosus between 13 and 17 weeks of gestation: Histological and morphometric studies. Ultrasound Obstet Gynecol 19: 39-46, 2002.

2. Kiserud T, Eik-Nes S, Blass HG and Hellevik LR: Ultrasonographic velocimetry of the fetal ductus venosus. Lancet 338: 1412-1414, 1991.

3. Hyett JA, Brizot ML, Von Kaisenberg C, Mckie AT, Farzanah F and Nicolaides KH: Cardiac gene expression of atrial natriuretic factorand brain natriuretic peptide in trisomic fetuses. Obstet Gynecol 87: 506-510, 1996.

4. Della Ragione F, Mastrovito P, Campanile C, Conti A, Papageorgiou EA, Hultén MA, Patsalis PC, Carter NP and D'Esposito M: Differential DNA methylation as a tool for noninvasive prenatal diagnosis (NIPD) of $\mathrm{X}$ chromosome aneuploidies. J Mol Diagn 12: 797-807, 2010.

5. Syngelaki A, Guerra L, Ceccacci I, Efeturk T and Nicolaides KH: Impact of holoprosencephaly, exomphalos, megacystis and high NTT in first trimester screening for chromosomal abnormalities. Ultrasound Obstet Gynecol 50: 45-48, 2017.

6. Vinkesteijn AS, Ursem NT, Struijk PC and Wladimiroff JW: Fetal heart rate and blood flow velocity variability in the presence of increased nuchal translucency: A preliminary study. Ultrasound Obstet Gynecol 23: 19-22, 2004.

7. Witters I and Fryns JR: Fetal nuchal translucency thickness. Genet Couns 18: 1-7, 2007.

8. Maiz N and Nicolaides KH: Ductus venosus in the first trimester: Contribution to screening of chromosomal, cardiac defects and monochorionic twin complications. Fetal Diagn Ther 28: 65-71, 2010.

9. Martínez JM, Comas M, Borrell A, Bennasar M, Gómez O, Puerto B and Gratacós E: Abnormal first-trimester ductus venosus blood flow: A marker of cardiac defects in fetuses with normal karyotype and nuchal translucency. Ultrasound Obstet Gynecol 35: 267-272, 2010.

10. Maiz N, Valencia C, Kagan KO, Wright D and Nicolaides KH: Ductus venosus Doppler in screening for trisomies 21, 18 and 13 and Turner syndrome at 11-13 weeks of gestation. Ultrasound Obstet Gynecol 33: 512-517, 2009.

11. Prefumo F, Sethna F, Sairam S, Bhide A and Thilaganathan B: First-trimester ductus venosus, nasal bones, and down syndrome in a high-risk population. Obstet Gynecol 105: 1348-1354, 2005.

12. Matias A, Gomes C, Flack N, Montenegro N and Nicolaides KH: Screening for chromosomal abnormalities at 10-14 weeks: The role of ductus venosus blood flow. Ultrasound Obstet Gynecol 12: 380-384, 1998
13. Hsiao CH, Cheng PJ, Shaw SW, Hsu JJ, Chen RC, Tseng YJ and Chu WC: Extended first-trimester screening using multiple sonographic markers and maternal serum biochemistry: A five-year prospective study. Fetal Diagn Ther 35: 296-301, 2014.

14. Jagielski J and Skawiński W: The analysis and classification of chromosomes. I. Application of the Bayes' theorem. Mater Med Pol 10: 198-203, 1978

15. Nikitin A, Egorov S, Daraselia N and Mazo I: Pathway studio-the analysis and navigation of molecular networks. Bioinformatics 19: 2155-2157, 2003.

16. Novichkova S, Egorov $\mathrm{S}$ and Daraselia N: MedScan, a natural language processing engine for MEDLINE abstracts. Bioinformatics 19: 1699-1706, 2003.

17. Harris LH: Rethinking maternal-fetal conflict: Gender and equality in perinatal ethics. Obstet Gynecol 96: 786-791, 2000.

18. Murta CG, Moron AF, Avila MA and Weiner CP: Application of ductus venosus Doppler velocimetry for the detection of fetal aneuploidyin the first trimester of pregnancy. Fetal Diagn Ther 17: 308-314, 2002.

19. Zoppi MA, Putzolu M, Ibba RM, Floris M and Monni G: First-trimester ductus venosus velocimetry in relation to nuchal translucency thickness and fetal karyotype. Fetal Diagn Ther 17: 52-57, 2002.

20. Borrell A, Martinez JM, Seres A, Borobio V, Cararach V and Fortuny A: Ductus venosus assessment at the time of nuchal translucency measurement in the detection of fetal aneuploidy. Prenat Diagn 23: 921-926, 2003.

21. Antolin E, Comas C, Torrents M, Muñoz A, Figueras F, Echevarria M, Cararach M and Carrera JM: The role of ductus venosus bloodflow assessment in screening for chromosomal abnormalities at 10-16 weeks of gestation. Ultrasound Obstet Gynecol 17: 295-300, 2001.

22. Toyama JM, Brizot ML, Liao AW, Lopes LM, Nomura RM, Saldanha FA and Zugaib M: Ductus venosus blood flow assessment at 11 to 14 weeks of gestation and fetal outcome. Ultrasound Obstet Gynecol 23: 341-345, 2004

23. Stressig R, Kozlowski P, Froehlich S, Siegmann HJ, Hammer R, Blumenstock $\mathrm{G}$ and Kagan KO: Assessment of the ductus venosus, tricuspid blood flow and the nasal bone in second-trimester screening for trisomy 21. Ultrasound Obstet Gynecol 37: 444-449, 2011

24. Wagner P, Sonek J, Hoopmann M, Abele H and Kagan KO: First-trimester screening for trisomies 18 and 13, triploidy and Turner syndrome by detailed early anomaly scan. Ultrasound Obstet Gynecol 48: 446-451, 2016.

25. Abdelmoez A, Coraça-Huber DC, Thurner GC, Debbage P, Lukas P, Skvortsov S and Skvortsova II: Screening and identification of molecular targets for cancer therapy. Cancer Lett 387: 3-9, 2017. 